
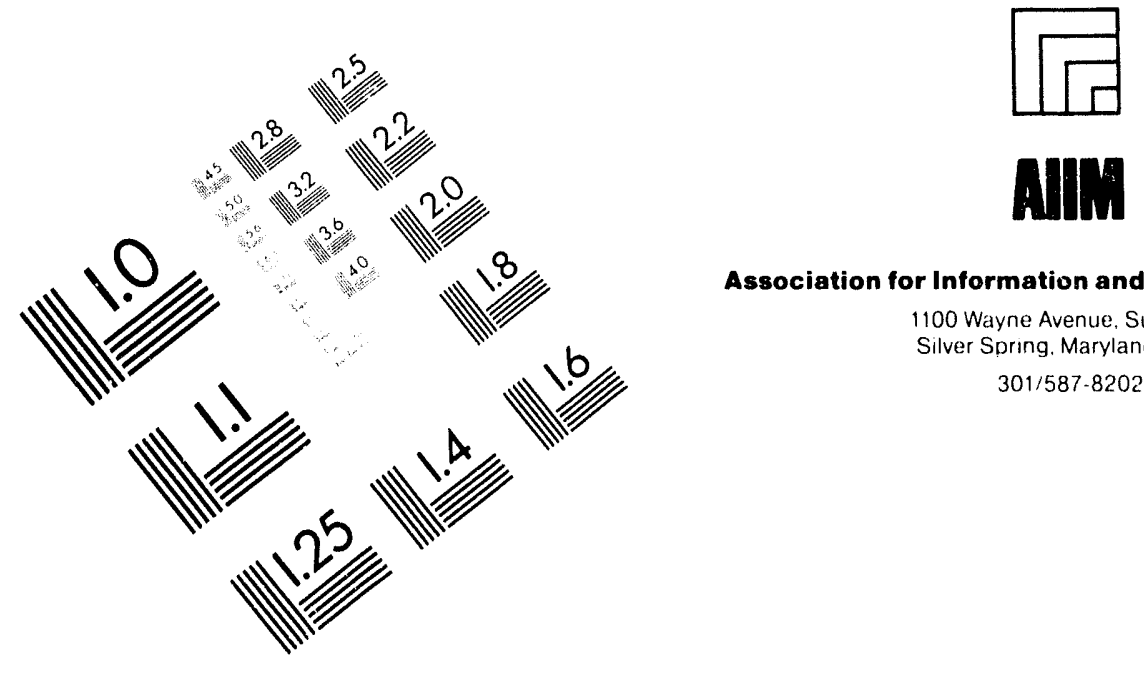

AlliM

Association for Information and Image Management

1100 Wayne Avenue. Sute 1100

Silver Spring. Maryland 20910

$301 / 587-8202$

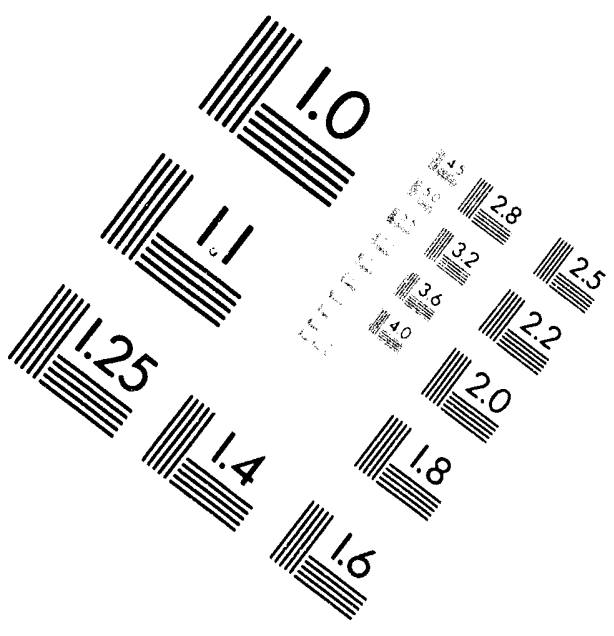

\title{
Centimeter
}

$\left.\right|_{1} ^{1}$

Inches
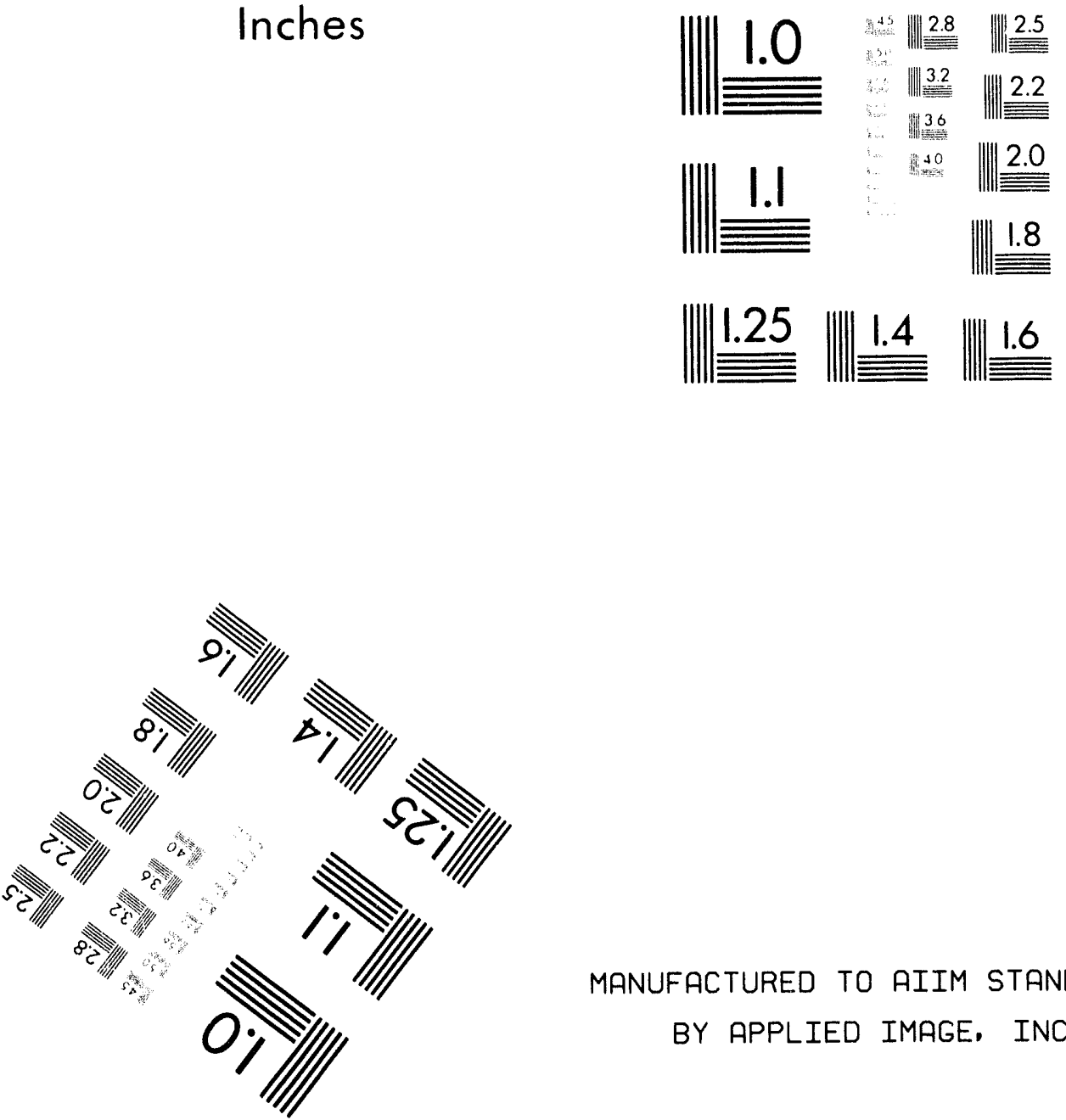

MANUFACTURED TO AIIM STANDARDS

BY APPLIED IMAGE, INC.

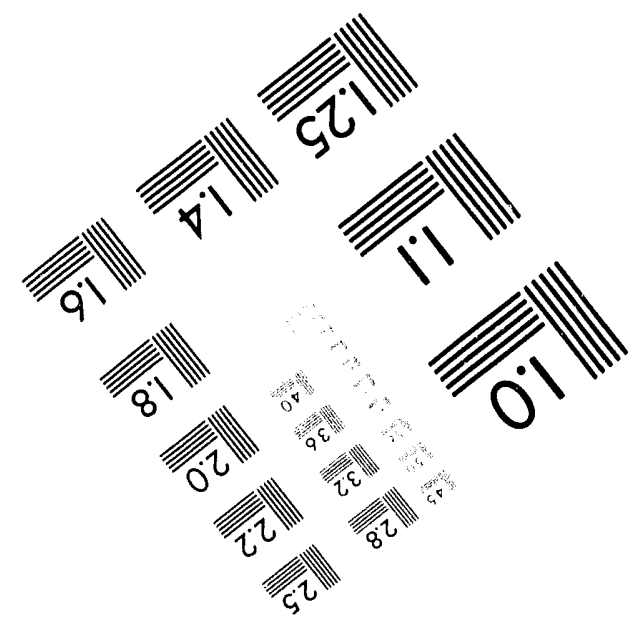



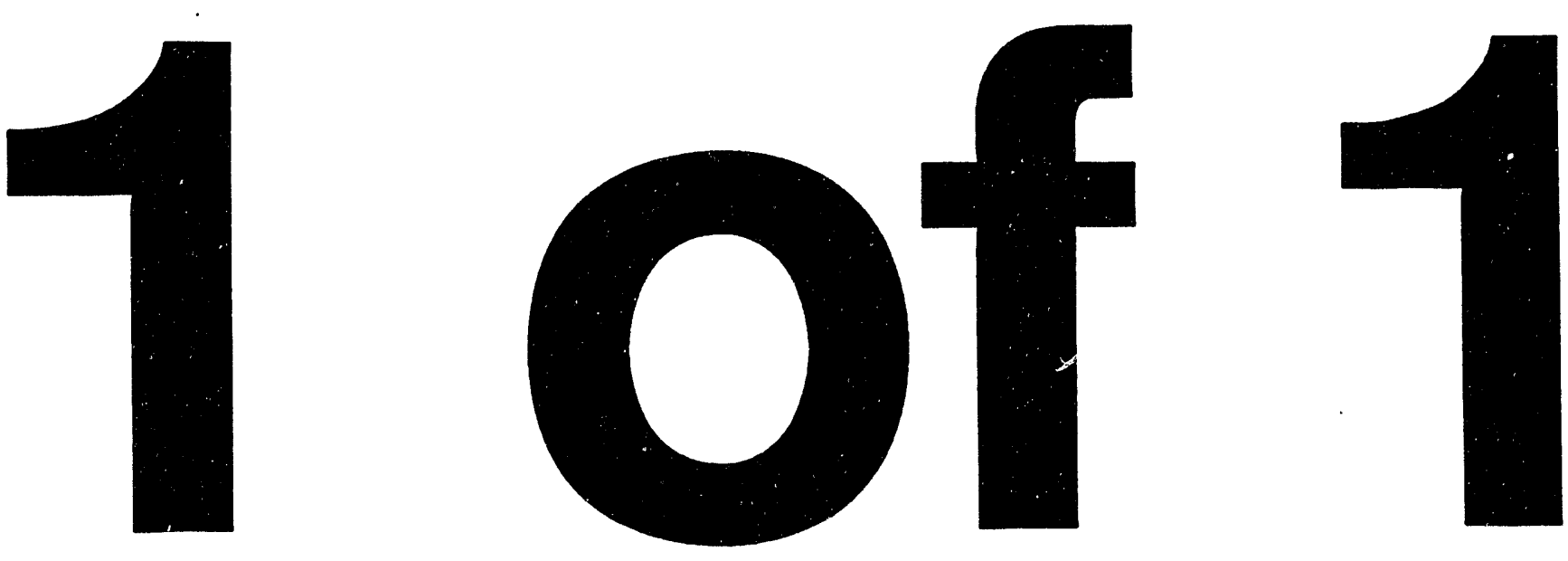
ORNL/TM-12757

Dist. Category UC-426

Fusion Energy Division

\section{FEEDBACK CONTROL AND STABILIZATION EXPERIMENTS ON THE TEXAS EXPERIMENTAL TOKAMAK (TEXT)}
T. Uckan
B. Richards*
P. D. Hurwitz*
A. J. Wootton*
P. E. Phillips*
R. D. Bengtson*
W. L. Rowan*
R. Bravenec*
H. Y. W. Tsui*
B. A. Carreras
J. R. Uglum*
G. X. $\mathrm{Li}^{*}$
Y. Wen*
D. Winslow*

*Fusion Research Center, The University of Texas at Austin, Austin, TX 78712.

This is a preprint of a paper presented at the 11 th International Conference on Plasma Surface Interactions in Controlled Fusion Devices, May 23-27, 1994, Ibaraki-ken, Japan.

Date Published: June 1994

Prepared for the

Office of Fusion Energy

Budget Activity No. AT 10

Prepared by

OAK RIDGE NATIONAL LABORATORY

Oak Ridge, Tennessee 37831

managed by

MARTIN MARIETTA ENERGY SYSTEMS, INC.

for the

U.S. DEPARTMENT OF ENERGY

under contract DE-AC05-84OR21400 


\section{CONTENTS}

\section{Page}

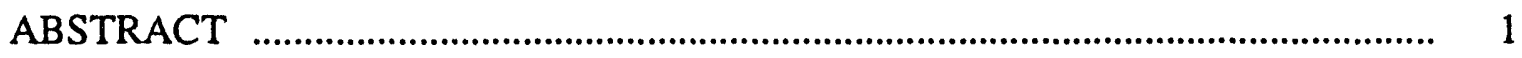

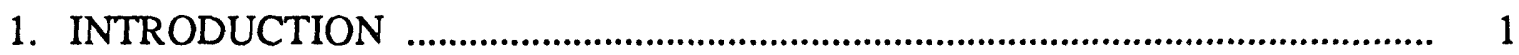

2. FEEDBACK WAVE-LAUNCHING SYSTEM AND DIAGNOSTIC .................. 2

3. EXPERIMENTAL OBSERVATIONS AND RESULTS ....................................... 4

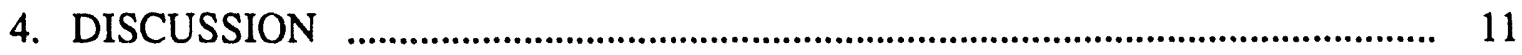

ACKNOWLEDGMENTS …...................................................................... 11

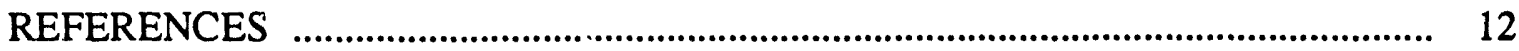




\title{
FEEDBACK CONTROL AND STABILIZATION EXPERIMENTS ON THE TEXAS EXPERIMENTAL TOKAMAK (TEXT)
}

\author{
T. Uckan \\ B. Richards \\ A. J. Wootton \\ R. D. Bengtson \\ R. Bravenec \\ B. A. Carreras \\ G. X. Li
}

\author{
P. D. Hurwitz \\ P. E. Phillips \\ W. L. Rowan \\ H. Y. W. Tsui \\ J. R. Uglum \\ Y. Wen \\ D. Winslow
}

\begin{abstract}
Plasma edge feedback experiments on the Texas Experimental Tokamak (TEXT) have been successful in controiling the edge plasma potential fluctuation level. The feedback wave-launcher, consisting of electrostatic probes located in the shadow of the limiter, is driven by the local edge potential fluctuations. In general, the edge potential fluctuations are modified in a broad frequency band. Moreover, it is observed that the potential fluctuations can be reduced $(\leq 100 \mathrm{kHz})$ without enhancing other modes, or excited (10 to $12 \mathrm{kHz}$ ), depending on the phase difference between the driver and the launcher signal, and gain of the system. This turbulence modification is achieved not only locally but also halfway around the torus and has about $2 \mathrm{~cm}$ of poloidal extent. Experiments on the characterization of the global plasma parameters with the edge feedback are discussed. Effects of the edge feedback on the estimated fluctuation-induced radial particle flux as well as on the local plasma parameters are presented.
\end{abstract}

\section{INTRODUCTION}

The edge plasma fluctuations in tokamaks play a significant role in the overall plasma confinement characteristics [1]. Experiments on the Texas Experimental Tokamak (TEXT) indicate that electrostatic fluctuations in the edge plasma are responsible for most of the energy and particle transport [2]. Although the underlying basic mechanisms that drive the edge turbulence are still the subject of ongoing research in fusion devices, in this work, a possible control and influence of the edge plasma fluctuation level are investigated on TEXT. These exploratory experiments have been performed utilizing edge plasma feedback to study its effects on edge turbulence characteristics as well as on the global plasma parameters. The local edge potential fluctuations are used to drive the feedback wave-launcher located in the shadow of the limiter. A number of earlier feedback 
experiments in various plasmas, that is, mirrors, Q-machines, and stellarators [3], have been designed to stabilize and suppress low-frequency instabilities. The overall observation in those experiments was that while suppression of the dominant lowest-order mode was possible, nevertheless, new, higher-order modes appeared $[3,4]$. In this report, the results observed from the recent edge feedback control and stabilization experiments performed on TEXT are summarized. Moreover, experimental measurements are presented on the broadband reduction of edge plasma fluctuations on a tokamak machine without enhancing new modes. In addition, observations on the effects of the edge feedback on local plasma parameters are discussed.

\section{FEEDBACK WAVE-LAUNCHING SYSTEM AND DIAGNOSTIC}

The edge feedback experiments are performed with a wave-launching system consisting of two electrostatic launching probes (L1, L2) shown in Fig. 1. These active probes (APs) are operated on the electron side of the (I,V) characteristic. This operating region, which is made by introducing a positive dc bias to the probes, provides a large dynamic response to the launcher as a result of the broad linear region available there. Each probe is fed separately by independent ac power supplies capable of providing up to $1.5 \mathrm{~kW}$ of power in the frequency range of 9 to $250 \mathrm{kHz}$. As shown in Fig. 1, the power sources are driven by a plasma signal through an isolated voltage divider and an adjustable frequency band pass filter, typically 10 to $100 \mathrm{kHz}$, with a voltage gain $(\mathrm{G})$ control, and then a unity gain variable phase shifter follows. The input sensing (driver) signal to the launcher can either be fluctuating density or plasma potential. The phase shifter provides an independent control of the ac phase difference $\Delta \varphi$ between the driver and the launching signals. To expect to have significant external influence on the edge turbulence, APs are poloidally separated by $d \sim \lambda / 2=1.8 \mathrm{~cm}$, with $\lambda=2 \pi / \bar{k}$, where $\bar{k}$ is the mean poloidal wavenumber of ine electrostatic fluctuations. The AP can handle an ac probe current of up to $\tilde{I}_{\mathrm{ac}} \sim 15 \mathrm{~A}$. An impedance matching (MATCH) (Fig. 1) network between the launcher and the power source is included for maximum power delivery to the plasma. On the launcher head, beside the APs there are two extra small sensors ( $\mathrm{S} 1, \mathrm{~S} 2$ ), as illustrated in Fig. 1, separated by $d / 2$, to measure the local plasma floating potential $\phi$. To reduce the power load on AP during the plasma discharge, the launcher head is placed on a pneumatic mechanism that provides the fast radial stroke. The fast plunging action into the plasma takes about $50 \mathrm{~ms}$ for a $5-\mathrm{cm}$ stroke; the probe stationary time is $\sim 100 \mathrm{~ms}$. The main diagnostic utilized during these experiments is a poloidally aligned seven-pin (these are $1 \mathrm{~cm}$ poloidally separated) sensor probe (SPSP) array for measuring $\phi$, located at the bottom center of the machine halfway around the torus from AP, separated by $\sim 157^{\circ}$ toroidally and $180^{\circ}$ poloidally, and used as a downstream sensing probe. 


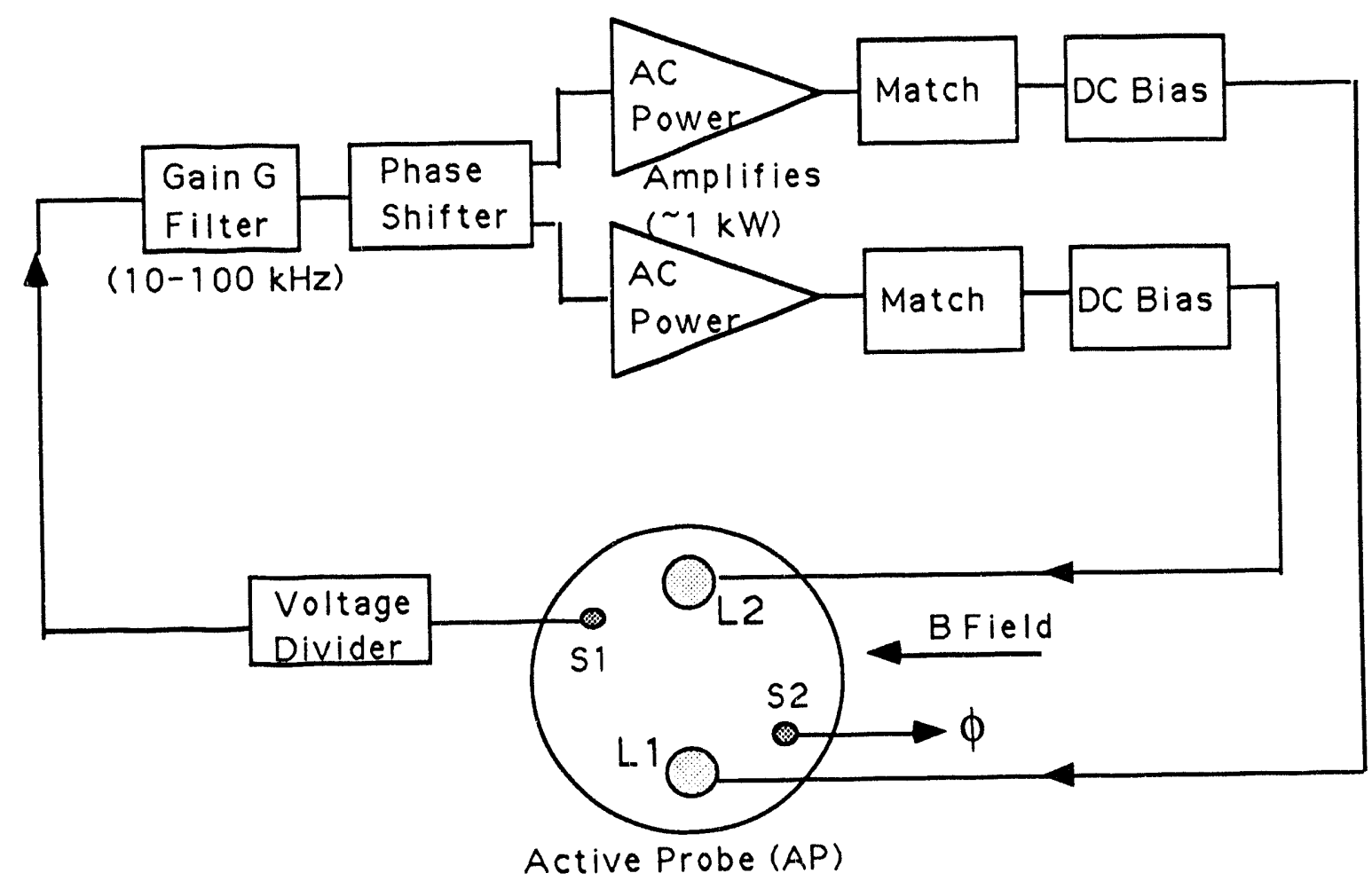

Fig. 1. Schematic of edge feedback wave-launching system, the active probe (AP). Launchers $(\mathrm{L} 1, \mathrm{~L} 2)$, which are oriented poloidally, are driven by local floating potential fluctuations $\tilde{\phi}$ obtained from sensor S1. Near-field (local) feedback effects are monitored with sensor S2. 


\section{EXPERIMENTAL OBSERVATIONS AND RESULTS}

The series of edge feedback experiments are performed in ohmically heated plasmas with a flat top of $\sim 300 \mathrm{~ms}$ in hydrogenic discharges. The toroidal magnetic field is $B \sim 2.2$ $\mathrm{T}$; the line average plasma density is $\bar{n}_{\mathrm{e}} \approx 3 \times 10^{13} \mathrm{~cm}^{-3}$. The rail limiters (top, bottom, and outside), are located at $r_{\mathrm{p}}=27 \mathrm{~cm}$; AP is at the limiter shadow, where $r_{\mathrm{ap}}=27.5 \mathrm{~cm}$, at the top center of the plasma column. The present feedback experiments are performed by driving the launcher system with the local floating potential fluctuations $\widetilde{\phi}$, obtained from the sensor $\mathrm{S} 1$ (see Fig. 1). The launcher dc bias of $\sim 50 \mathrm{~V}$ is used. Results of comparison experiments with and without the applied dc bias, which is about one-third of the local plasma potential, indicate that this dc bias is not enough to otherwise affect the results of the feedback experiments. As observed in earlier limiter biasing experiments [5] performed with $\sim 50 \mathrm{~V}$, there was almost no effect on the global and turbulence characteristics of the edge plasma.

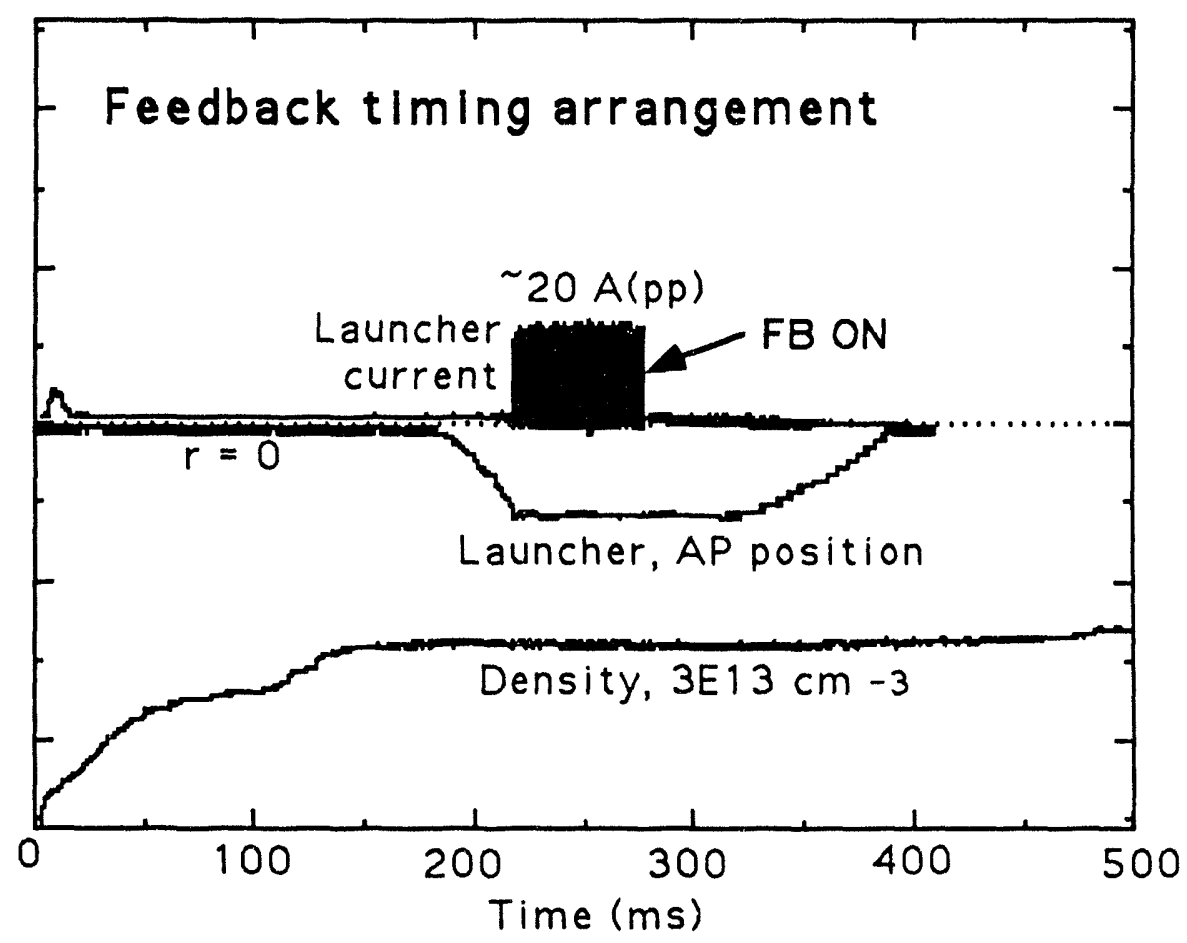

Fig. 2. Feedback arrangement during plasma discharge. When AP reaches its final radial position $r_{\text {app }}$, feedback is then applied (FB on) for $\sim 40 \mathrm{~ms}$ during flat top of the discharge.

As shown in Fig. 2, when AP reaches its set final radial position, the edge feedback is then applied [feedback (FB) on] for $\sim 40 \mathrm{~ms}$ during the flat top of the plasma discharge. The results are compared to the measurements that are taken when the feedback is off (FB off) during the same discharge. The results of the earlier wave-launching and excitation experiments $[6,7]$, that is, injecting $\sim 30-\mathrm{kHz}$ signal at the edge, have indicated that the 
propagation is taking place along the magnetic field line. Therefore, these feedback experiments are performed by locating AP, the wave-launcher, and the downstream sensing probe SPSP, which acts as a far-field sensor, on the same magnetic field line. This configuration is obtained from detailed calculations of the magnetics of TEXT, which is possible with a plasma current of $I_{\mathrm{p}} \sim 180 \mathrm{kA}$ at $r=27.5 \mathrm{~cm}$.

The following experiments are carried out with a typical wave-launcher ac current of $\tilde{I}_{\mathrm{ac}} \sim 5 \mathrm{~A}$ (for the gain setting of $\mathrm{G}=10$ ) which may be viewed as the minimum value for the experiment. The observed results of the edge feedback on the spectrum of the potential fluctuations (FFT of $\tilde{\phi}$ ) are summarized in Figs. 3 and 4, for $\Delta \varphi=0$ and $\pi$, respectively; the no feedback case is also included for comparison. Here, Figs. 3(a) and 4(a) represent the local effects, measured with the sensing tip S2 (Fig. 1), the near-field sensor. On the other hand, Figs. 3(b) and 4(b), which are obtained from the measurements of SPSP, the far-field sensor, show the observed effects about $12 \mathrm{~m}$ away from where the edge feedback is introduced.

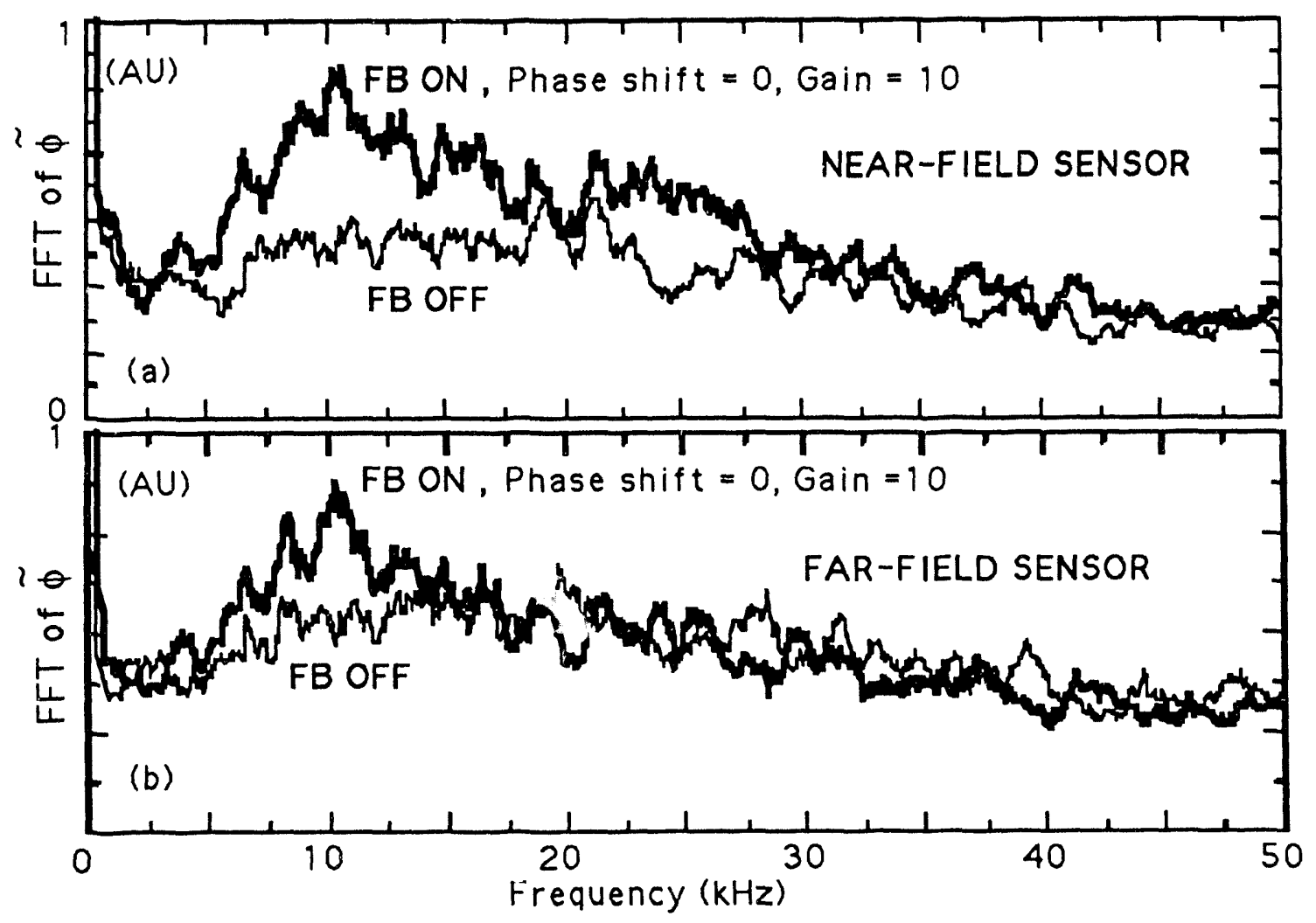

Fig. 3. Effects of edge feedback $(G=10)$ on frequency spectrum of potential fluctuations (FFT of $\tilde{\phi}$ ) measured at the location of (a) AP (near-field effects from S2), and (b) SPSP (far-field effects) for the phase shift setting of $\Delta \varphi=0$ compared to no feedback case. 


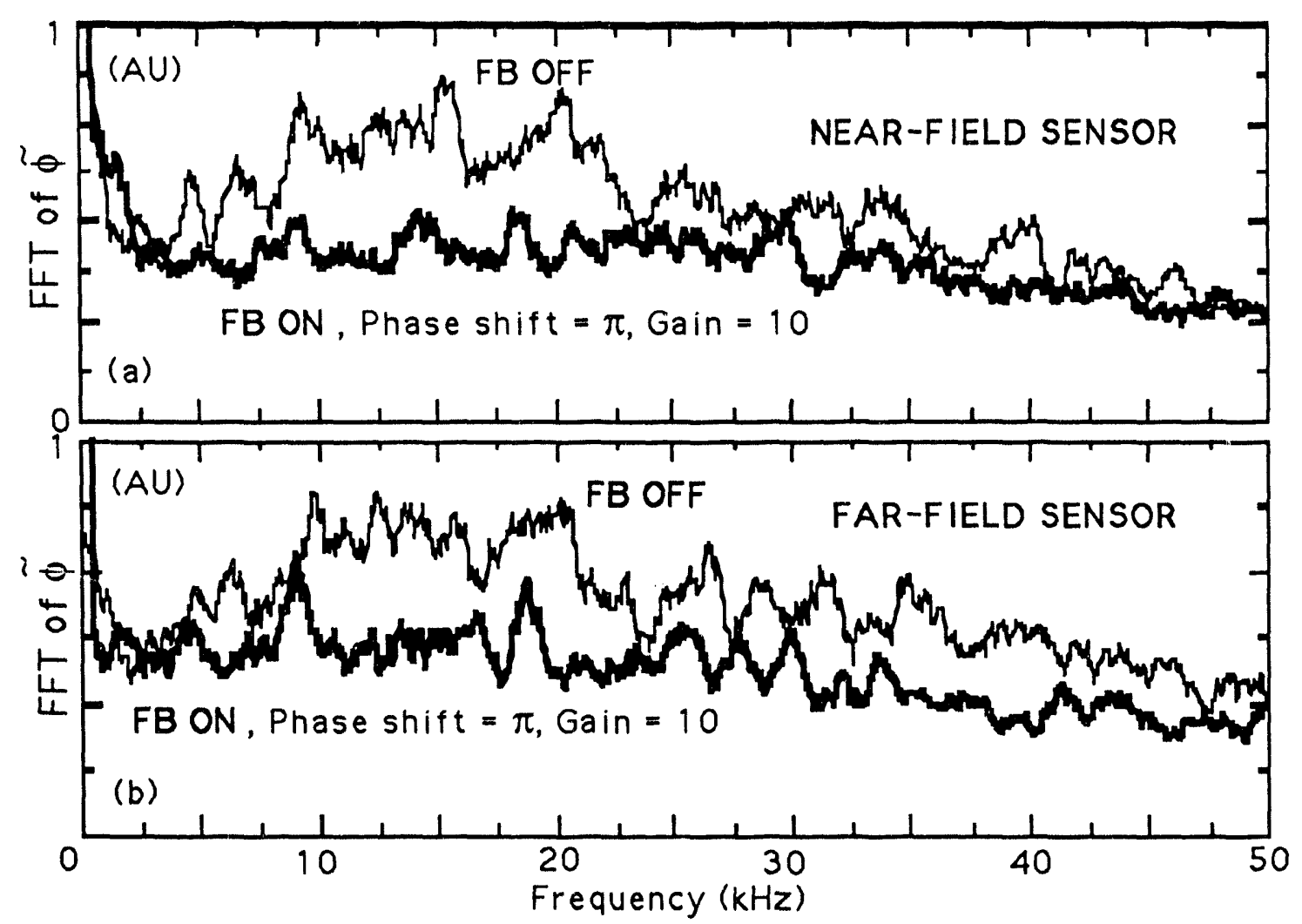

Fig. 4. Same measurements as in Fig. 3, but for $\Delta \varphi=\pi$.

In general, as discussed in detail in ref. [7], a broadband frequency modification of the edge potential fluctuations, which are the dominant edge fluctuations in TEXT [8], is achieved. Moreover, it is observed that the edge potential fluctuations can be reduced ( $\leq 100-\mathrm{kHz}$ ) negative feedback (stabilization), $\Delta \varphi=\pi$, without enhancing other modes, or excited $(\sim 10-\mathrm{kHz})$ positive feedback (destabilization), $\Delta \varphi=0$, depending on $\Delta \varphi$, both the near-field and at the location of the far-field sensing probe SPSP. The $f_{\mathrm{mhd}} \sim 9.5 \mathrm{-kHz}$ peak seen in Fig. 4, with the feedback, is identified to be the MHD activity. 


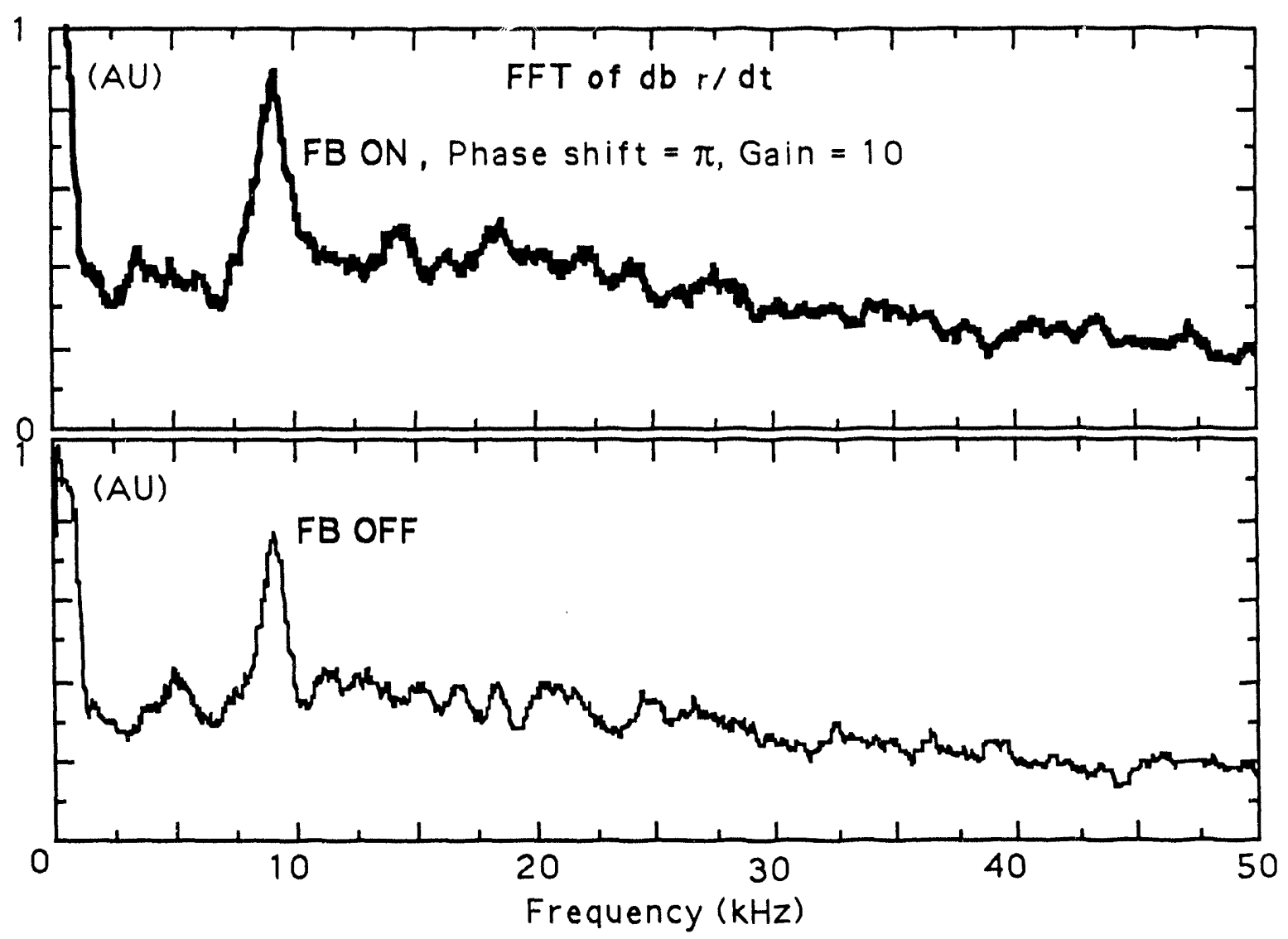

Fig. 5. Effects of edge feedback on MHD signal (FFT of $\dot{b}_{\mathrm{r}}$ ) for $\Delta \varphi=\pi$.

In Fig. 5, the effects of the edge feedback observed from one of the MHD coils, which is located at the top of the machine $\left(r>r_{\mathrm{p}}\right)$ and oriented to sense the radial component of the fluctuating magnetic field $\dot{b}_{\mathrm{r}}$, is presented for $\Delta \varphi=\pi$. The $f_{\mathrm{mhd}} \sim 9.5 \mathrm{kHz}$ activity is somewhat enhanced by about $15 \%$, and the rms value of $\dot{b}_{\mathrm{r}}$ is $\sim 12 \%$ higher with the feedback. In the case of $\Delta \varphi=0$, there is no significant change on $f_{\mathrm{mhd}}$ while the rms value of $\dot{b}_{\mathrm{r}}$ is $\sim 15 \%$ higher. 


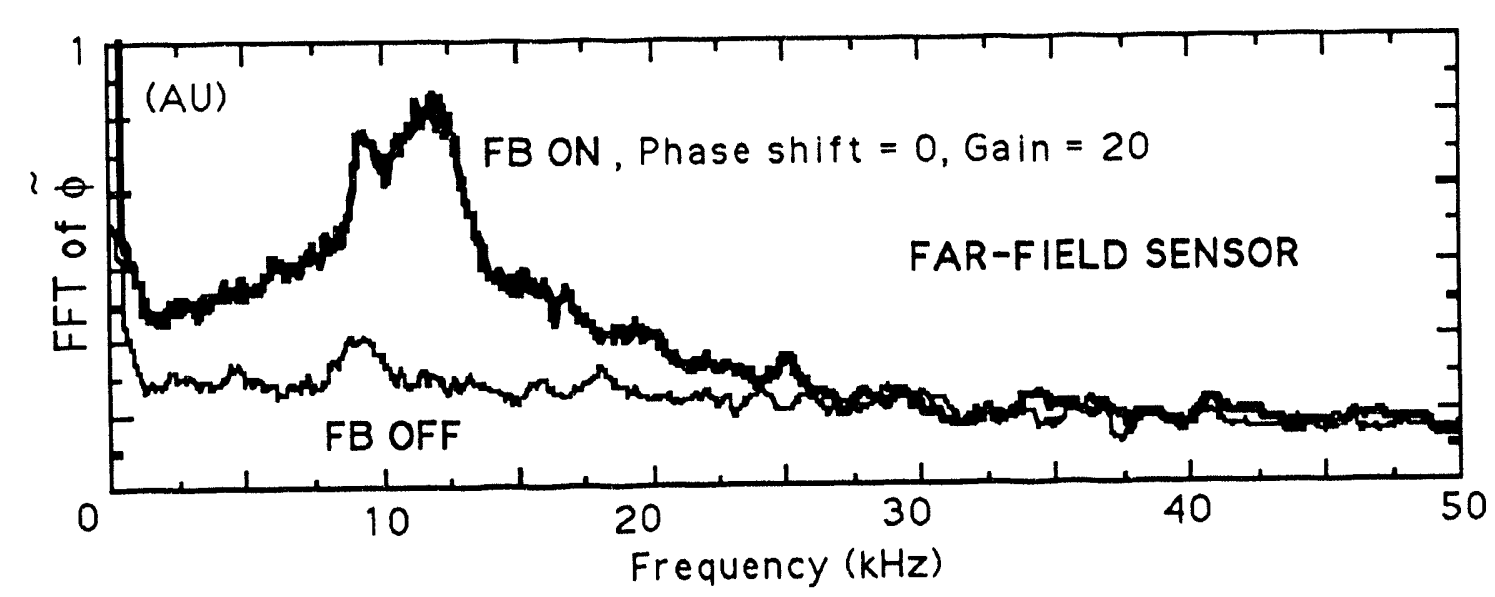

Fig. 6. Effects of edge feedback on spectrum of potential fluctuations measured at far-field sensor SPSP for $\Delta \varphi=0$ and $G=20$ compared to no feedback case.

In the next experiment the feedback gain is increased by a factor of $2(G=20)$, and the resulting spectrum of $\tilde{\phi}$ in the far-field sensor is given in Fig. 6 for $\Delta \varphi=0$. In this case $\tilde{I}_{\mathrm{ac}} \sim 10 \mathrm{~A}$ is causing a significant, by about a factor of 3 , destabilization (excitations) near $\sim 12 \mathrm{kHz}$. At the same time $\tilde{\phi}$ level around $f_{\mathrm{mhd}} \sim 9.5-\mathrm{kHz}$ (Fig. 6) is also increased by about a factor of 2 .

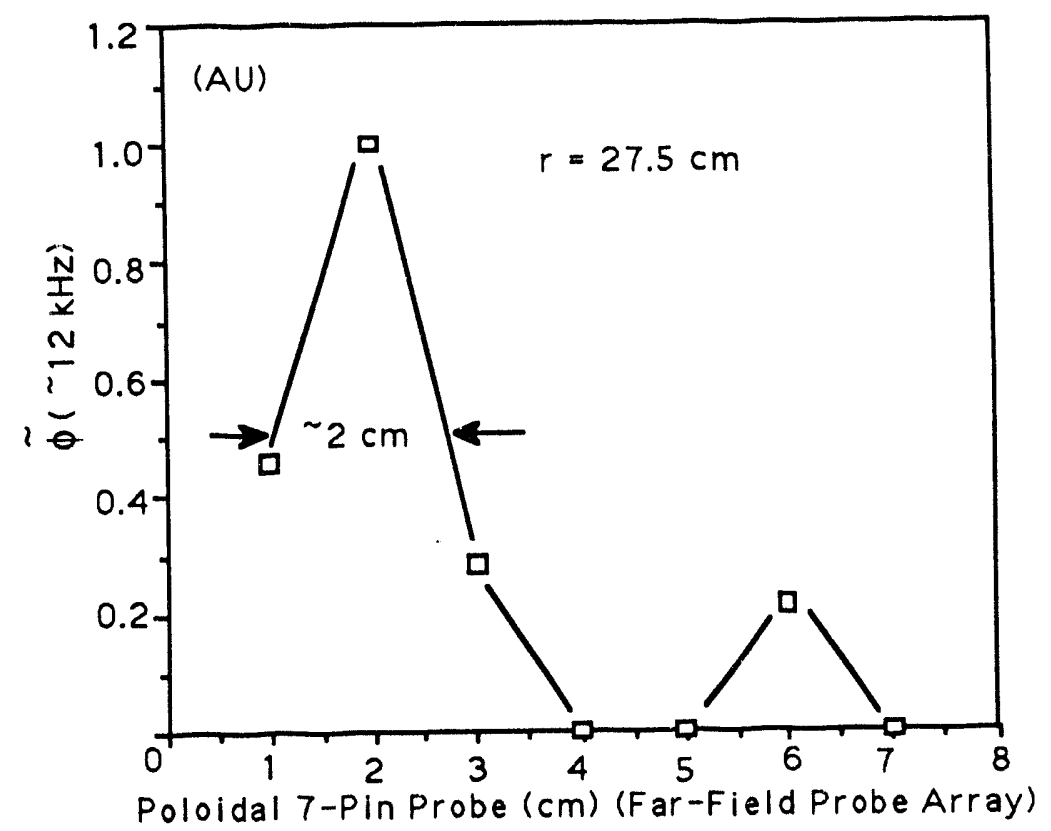

Fig. 7. Poloidal profile of feedback excitation [FFT of $\tilde{\phi}(\sim 12 \mathrm{kHz})$ with respect to no feedback case] obtained by 7-pin poloidally aligned sensor probe array (pins are $1 \mathrm{~cm}$ poloidally separated) located at $r=27.5 \mathrm{~cm}$; it is $\sim 12 \mathrm{~m}$ away from AP. Typical measured poloidal extent is about $2 \mathrm{~cm}$ (FWHM). 
In addition, the poloidal extent of the feedback excitations on $\tilde{\phi}$ is investigated. The measurements with SPSP give about $2 \mathrm{~cm}$ (FWHM) poloidal extent (Fig. 7) for the feedback excitation of $\tilde{\phi}$. These results indicate that the edge feedback can modify the amplitude of the potential fluctuations effectively not only locally but also halfway around the torus along a magnetic field line.

Previous edge feedback experiments [6] (with $G=10$ ) carried out with a fast reciprocating Langmuir probe (FRLP) array [9], located at the same port as SPSP, gave similar results, but only in low frequencies $(\leq 10 \mathrm{kHz})$, on the fluctuating ion saturation current, which is mostly dominated by the local density fluctuations $\tilde{n}$. The results of those feedback experiments in terms of the phase shift $\Delta \varphi_{12}$ between the probes L1 and L2 with respect to $\mathrm{L} 1$ on the rms values of the potential and the density fluctuations, normalized to their values without the feedback, are given in Fig. 8.

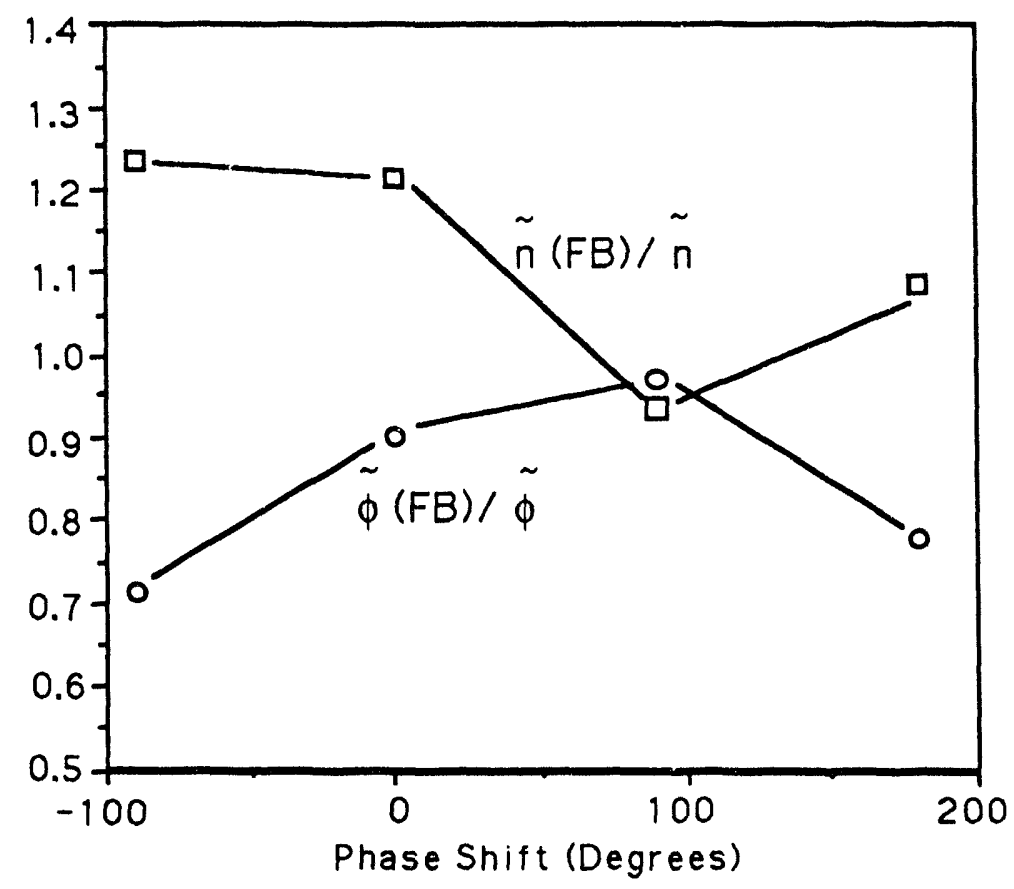

Fig. 8. Effects of feedback (FB) in terms of phase shift between probes $\mathrm{L} 1$ and $\mathrm{L} 2$ on rms values of potential and density fluctuations, normalized to without feedback.

This figure shows that the present feedback scheme appears to be more effective for reducing the edge potential fluctuations than the density fluctuations, due to broadband reduction of the potential fluctuations with the edge feedback. The corresponding fluctuation-induced radial particle flux $\widetilde{\Gamma}_{\mathrm{r}}$ is also estimated by ignoring the temperature fluctuations [8]. Using the measurements of FRLP, the estimated $\widetilde{\Gamma}_{\mathrm{r}}$ is somewhat modified by the edge feedback. For example, $\widetilde{\Gamma}_{\mathrm{r}}$ is $\sim 20 \%$ higher than without the feedback when $\Delta \varphi_{12}=0$, but it becomes $\sim 30 \%$ lower when $\Delta \varphi_{12}=\pi / 2$. 
The effects of the edge feedback on the frequency spectrum of the edge bolometer, $\mathrm{r} \sim 27 \mathrm{~cm}$, signal $\mathrm{P}_{\mathrm{rad}}$, which is related to the impurity radiation integrated along the line-of-sight of the detector, is shown in Fig. 9 for $\Delta \varphi=0$. This signal is related to $\mathrm{P}_{\mathrm{rad}} \sim n_{\mathrm{e}} n_{\mathrm{Z}} \mathrm{I}_{\mathrm{Z}}\left(T_{\mathrm{e}}\right)$, where $n_{\mathrm{Z}}$ is the impurity density, and $\mathrm{I}_{\mathrm{Z}}\left(T_{\mathrm{e}}\right)$ represents the impurity radiation cooling rate for the edge electron temperature $T_{\mathrm{e}}$.

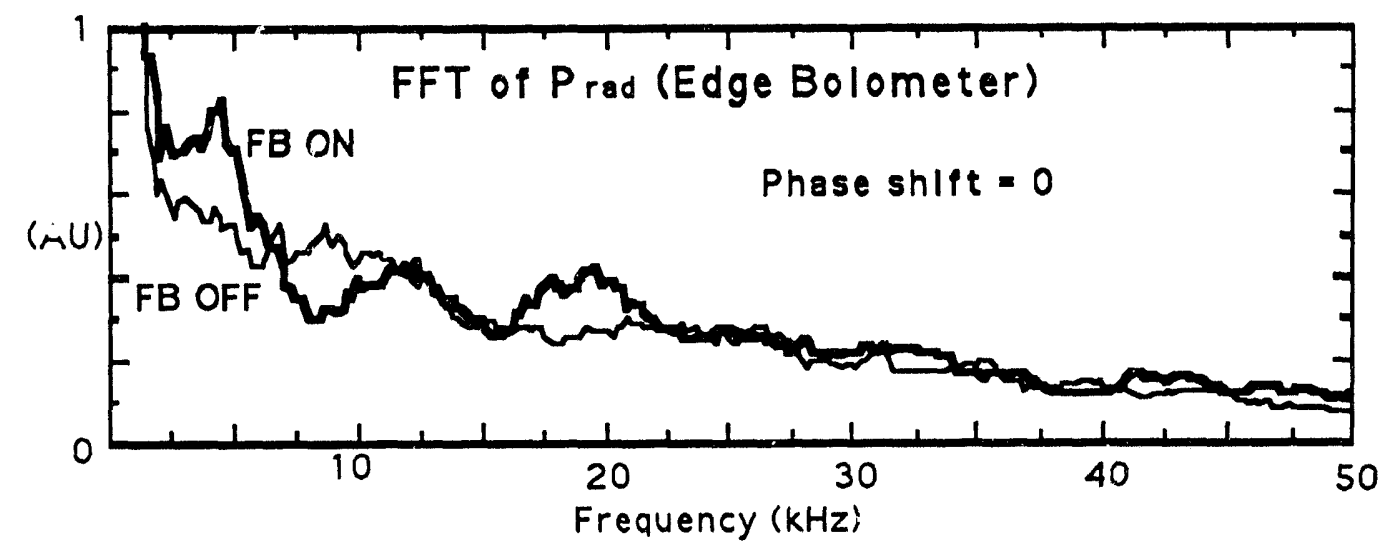

Fig. 9. Frequency spectrum of edge bolometer signal is shown with edge feedback for $\Delta \varphi=0$.

Thus, the data of Fig. 9 indicates that the edge plasma parameters are somewhat modified by the edge feedback. For example, the rms value of the fluctuating bolometer signal is $\sim 20 \%$ higher than without the feedback. The radial edge plasma density and temperature profiles are not available at this time, but their local values are observed to be affected by the feedback. The measurements from FRLP, located ai $r=27.5 \mathrm{~cm}$, show that reduced $\tilde{\phi}$ leads to somewhat lower $T_{\mathrm{e}}$ and higher $n_{\mathrm{e}}$. For example, when $\tilde{\phi}$ is reduced as much as by about a factor of 0.7 by the feedback (Fig. 8), the measured local $T_{\mathrm{e}}$ is somewhat lower by about a factor of 0.75 . On the other hand, the corresponding edge density $n_{\mathrm{e}}$ is higher by about a factor of 1.4. Overall, it appears that a reduction of the edge potential fluctuations by the feedback results in lower local electron temperatures, as is observed in earlier studies on the turbulence scaling experiments on TEXT [10], and that, in turn, may cause the increase in the local density because the plasma pressure remains constant. This result may suggest a possible application of the edge feedback for controlling the edge power loads, $T \mathrm{e}^{3 / 2}$, to the plasma facing components. Moreover, the lifetime of the edge components can also be prolonged because the sputtering yield is lower due to reduced impact energy of ions, $\sim 5 T_{\mathrm{e}}[11]$, as a consequence of low $T_{\mathrm{e}}$ from the edge feedback. Meanwhile the global core plasma parameters have not been affected by the present feedback experiments. 


\section{DISCUSSION}

Plasma edge feedback experiments on TEXT have been successful in stabilizing the edge plasma potential fluctuation levels. Although the underlying physics issues have not been resolved from the present data, these experiments have demonstrated the feasibility of the edge feedback to influence, modify, and control the levels of the electrostatic edge fluctuations. Moreover, effects of the edge feedback on the local plasma parameters suggest the use of the feedback control to possibly influence the power levels on the edge components. Specifically, reduction of the electron temperature with the edge feedback lowers the erosion rate of the plasma facing components and, in turn, impurity production at the edge. On the basis of these observations, detailed experiments are planned with additional diagnostics at various feedback gain and phase shift settings for TEXT Upgrade, which has a divertor configuration. In addition, plans include measuring profiles with the optimized feedback schemes. These detailed experiments are needed for understanding the underlying mechanism of the edge feedback to design techniques for an effective control. More launchers will also be implemented to improve the poloidal flux coverage at the edge so that most impact on the global plasma characteristics can be achieved with the feedback.

\section{ACKNOWLEDGMENTS}

The authors thank the support of the TEXT group and operating staff. Special thanks are due to Dr. A. Sen, Columbia University, for his useful advice on the feedback experiments, and also to D. B. Crockett, K. W. Gentle, K. R. Carter, G. R. Dyer, and D. Patterson for their help in obtaining the hardware as weli as in making the experiment possible. One of the authors (TU) thanks to J. N. Leboeuf, and P. K. Mioduszewski for valuable discussions and continuous encouragement. 


\section{REFERENCES}

[1] A. J. Wootton et al., Plasma Physics and Controlled Fusion 34 (1992) 2023.

[2] Ch. P. Ritz et al., Phys. Rev. Lett. 62 (1989) 1844.

[3] T. K. Chu, and H. W. Hendel (Eds.), Feedback and Dynamic Control of Plasmas (American Inst. of Physics, New York, N. Y. 1970), p. 114.

[4] K. I. Thomassen et al., Nucl. Fusion 11 (1971) 175.

[5] P. E. Phillips et al., J. Nucl. Mater. 145 \& 147 (1987) 807.

[6] T. Uckan, B. Richards, R. D. Bengtson et al., in Proc. of the 20th EPS Conference on Controlled Fusion and Plasma Physics (1993) Vol. 17C, p. 635.

[7] B. Richards, T. Uckan, A. J. Wootton et al., Phys. Plasmas 1 (1994) 1606.

[8] Ch. P. Ritz et al., Nucl. Fusion 27 (1987) 1125.

[9] T. L. Rhodes et al., Rev. Sci. Instrum. 61 (1990) 3001.

[10] T. L. Rhodes et al., Nucl. Fusion 33 (1993) 1147.

[11] P. C. Stangeby and G. M. McCracken, Nucl. Fusion 30 (1990) 1225. 
ORNL/TM-12757

Dist. Category UC-426

\section{INTERNAL DISTRIBUTION}

$\begin{aligned} \text { 1. } & \text { Director, Fusion Energy Division } \\ 2 . & \text { L. A. Berry } \\ 3-7 . & \text { B. A. Carreras } \\ 8 . & \text { R. A. Dory } \\ 9 . & \text { T. E. Shannon } \\ \text {-17. } & \text { T. Uckan } \\ \text { 18. } & \text { L. A. Charlton } \\ \text { 19. } & \text { R. J. Colchin } \\ 20 . & \text { J. H. Harris } \\ 21 . & \text { R. C. Isler } \\ 22 . & \text { J. N. Leboeuf }\end{aligned}$
23. J. F. Lyon
24. P. K. Mioduszewski
25. M. Murakami
26. K. C. Shaing
27-28. Laboratory Records Department
29. Laboratory Records, ORNL-RC
30. Central Research Library
31. Document Reference Section
32. Fusion Energy Division Library
33. ORNL Patent Section

\section{EXTERNAL DISTRIBUTION}

34. Office of the Assistant Manager for Energy Research and Development, U.S. Department of Energy, ORO, Oak Ridge, TN 37831

35. J. D. Callen, Department of Nuclear Engineering, University of Wisconsin, Madison, WI 53706-1687

36. R. W. Conn, Department of Chemical, Nuclear, and Thermal Engineering, University of California, Los Angeles, CA 90024

37. N. A. Davies, Director, Office of Fusion Energy, Office of Energy Research, ER-50 Germantown, U.S. Department of Energy, Washington, DC 20545

38. S. O. Dean, Fusion Power Associates, Inc., 2 Professional Drive, Suite 248, Gaithersburg, MD 20879

39. R. W. Gould, Department of Applied Physics, California Institute of Technology, Pasadena, CA 91125

40. R. A. Gross, Plasma Research Laboratory, Columbia University, New York, NY 10027

41. D. M. Meade, Princeton Plasma Physics Laboratory, P.O. Box 451, Princeton, NJ 08543

42. M. Roberts, International Programs, Office of Fusion Energy, Office of Energy Research, ER-52 Germantown, U.S. Department of Energy, Washington, DC 20545

43. W. M. Stacey, School of Nuclear Engineering and Health Physics, Georgia Institute of Technology, Atlanta, GA 30332

44. D. Steiner, Nuclear Engineering Department, NES Building, Tibbetts Avenue, Rensselaer Polytechnic Institute, Troy, NY 12181

45. R. Varma, Physical Research Laboratory, Navrangpura, Ahmedabad 380009, India

46. Bibliothek, Max-Planck Institut für Plasmaphysik, Boltzmannstrasse 2, D-8046 Garching, Federal Republic of Germany

47. Bibliothek, Institut für Plasmaphysik, KFA Jülich GmbH, Postfach 1913, D-5170 Jülich, Federal Republic of Germany

48. Bibliothek, KfK Karlsruhe GmbH, Postfach 3640, D-7500 Karlsruhe 1, Federal Republic of Germany

49. Bibliothèque, Centre de Recherches en Physique des Plasmas, Ecole Polytechnique Fédérale de Lausanne, 21 Avenue des Bains, CH-1007 Lausanne, Switzerland

50. L. Laurent, CEN/Cadarache, Departement de Recherches sur la Fusion Contrôlée, F-13108 Saint-Paullez-Durance Cedex, France

51. Bibliothèque, CEN/Cadarache, F-13108 Saint-Paul-lez-Durance Cedex, France

52. Library, AEA Fusion, Culham Laboratory, Abingdon, Oxfordshire, OX14 3DB, England

53. Library, JET Joint Undertaking, Abingdon, Oxfordshire OX14 3EA, England 
54. Library, FOM-Instituut voor Plasmafysica, Rijnhuizen, Edisonbaan 14, 3439 MN Nieuwegein, The Netherlands

55. Library, National Institute for Fusion Science, Chikusa-ku, Nagoya 464-01, Japan

56. Library, International Centre for Theoretical Physics, P.O. Box 586, I-34100 Trieste, Italy

57. Library, Centro Ricerche Energia Frascati, C.P. 65, I-00044 Frascati (Roma), Italy

58. Library, Plasma Physics Laboratory, Kyoto University, Gokasho, Uji, Kyoto 611, Japan

59. Plasma Research Laboratory, Australian National University, P.O. Box 4, Canberra, A.C.T. 2601, Australia

60. Library, Japan Atomic Energy Research Institute, Naka Fusion Research Establishment, 801-1 Mukoyama, Naka-machi, Naka-gun, Ibaraki-ken, Japan

61. G. A. Eliseev, I. V. Kurchatov Institute of Atomic Energy, P.O. Box 3402, 123182 Moscow, Russia

62. V. A. Glukhikh, Scientific-Research Institute of Electro-Physical Apparatus, 188631 Leningrad, Russia

63. I. Shpigel, Institute of General Physics, U.S.S.R. Academy of Sciences, Ulitsa Vavilova 38, Moscow, Russia

64. D. D. Ryutov, Institute of Nuclear Physics, Siberian Branch of the Academy of Sciences, Sovetskaya St. 5, 630090 Novosibirsk, Russia

55. O. Pavlichenko, Kharkov Physical-Technical Institute, Academical St. 1, 310108 Kharkov, Ukraine

66. Deputy Director, Southwestern Institute of Physics, P.O. Box 15, Leshan, Sichuan, China (PRC)

67. Director, The Institute of Plasma Physics, P.O. Box 26, Hefei, Anhui, China (PRC)

68. R. A. Blanken, Experimental Plasma Physics Research Branch, Division of Applied Plasma Physics, Office of Energy Research, ER-542, Germantown, U.S. Deprrtment of Energy, Washington, DC 20545

69. R. A. E. Bolton, IREQ Hydro-Quebec Research Institute, 1800 Montée-Ste.-Julie, Varennes, P.Q. JOL 2P0, Canada

70. D. H. Crandall, Experimental Plasma Physics Research Branch, Division of Applied Plasma Physics, Office of Energy Research, ER-542, Germantown, U.S. Department of Energy, Washington, DC 20545

71. R. L. Freeman, General Atomics, P.O. Box 85608, San Diego, CA 92138-5608

72. K. W. Gentle, RLM 11.222, Institute for Fusion Studies, University of Texas, Austin, TX 78712

73. R. J. Goldston, Princeton Plasma Physics Laboratory, P.O. Box 451, Princeton, NJ 08543

74. J. C. Hosea, Princeton Plasma Physics Laboratory, P.O. Box 451, Princeton, NJ 08543

75. D. Markevich, Division of Confinement Systems, Office of Energy Research, ER-55, Germantown, U.S. Department of Energy, Washington, DC 20545

76. R. H. McKnight, Experimental Plasma Physics Research Branch, Division of Applied Plasma Physics, Office of Energy Research, ER-542, Germantown, U.S. Department of Energy, Washington, DC 20545

77. E. Oktay, Division of Confinement Systems, Office of Energy Research, ER-55, Germantown, U.S. Department of Energy, Washington, DC 20545

78. W. L. Sadowski, Fusion Theory and Computer Services Branch, Division of Applied Plasma Physics, Office of Energy Research, ER-541, Germantown, U.S. Department of Energy, Washington, DC 20545

79. J. W. Willis, Division of Confinement Systems, Office of Energy Research, ER-55, Germantown, U.S. Department of Energy, Washington, DC 20545

80. C. Alejaldre Division de Fusion, CIEMAT, Avenida Complutense 22, E-28040 Madrid, Spain

81. Laboratory for Plasma and Fusion Studies, Department of Nuclear Engineering, Seoul National University, Shinrim-dong, Gwanak-ku, Seoul 151, Korea

82. J. L. Johnson, Plasma Physics Laboratory, Princeton University, P.O. Box 451, Princeton, NJ 08543

83. L. M. Kovrizhnykh, Institute of General Physics, Russia Academy of Sciences, Ulitsa Vavilova 38, 117924 Moscow, Russia

84. O. Motojima, National Institute for Fusion Science, Chikusa-ku, Nagoya 464-01, Japan

85. S. Okamura, Institute of Plasma Physics, Nagoya University, Chikusa-ku, Nagoya 464, Japan

86. V. D. Shafranov, I. V. Kurchatov Institute of Atomic Energy, P.O. Box 3402, 123182 Moscow, Russia

87. J. L. Shohet, Torsatron/Stellarator Laboratory, University of Wisconsin, Madison, WI 53706

88. H. Wobig, Max-Planck Institut für Plasmaphysik, D-8046 Garching, Germany

89. F. S. B. Anderson, University of Wisconsin, Madison, WI 53706

90. R. F. Gandy, Physics Department, Auburn University, Auburn, AL 36849-3511

91. H. Kaneko, Plasma Physics Laboratory, Kyoto University, Gokasho, Uji, Japan

92. G. H. Neilson, Princeton Plasma Physics Laboratory, P.O. Box 451, Princeton, NJ 08543 
93. S. Sudo, Plasma Physics Laboratory, Kyoto University, Gokasho, Uji, Japan

94. H. Yamada, National Institute for Fusion Science, Chikusa-ku, Nagoya 464-01, Japan

95. F. W. Perkins, Princeton Plas.na Physics Laboratory, P.O. Box 451, Princeton, NJ 08543

96. T. Obiki, Plasma Physics Laboratory, Kyoto University, Gokasho, Uji, Kyoto, Japan

97. A. Iiyoshi, National Institute for Fusion Studies, Chikusa-ku, Nagoya 464-01, Japan

98. B. Richards, Fusion Research Center, University of Texas, Austin, TX 78712

99. R. D. Bengtson, Fusion Research Center, University of Texas, Austin, TX 78712

100. R. Bravenec, Fusion Research Center, University of Texas, Austin, TX 78712

101. G. X. Li, Fusion Research Center, University of Texas, Austin, TX 78712

102. P. D. Hurwitz, Fusion Research Center, University of Texas, Austin, TX 78712

103. P. E. Phillips, Fusion Research Center, University of Texas, Austin, TX 78712

104. W. L. Rowan, Fusion Research Center, University of Texas, Austin, TX 78712

105. H. Y. W. Tsui, Fusion Research Center, University of Texas, Austin, TX 78712

106. J. Uglum, Fusion Research Center, University of Texas, Austin, TX 78712

107. Y. Wen, Fusion Research Center, University of Texas, Austin, TX 78712

108. D. Winslow, Fusion Research Center, University of Texas, Austin, TX 78712

109. A. J. Wootton, Fusion Research Center, University of Texas, Austin, TX 78712

110-111. Office of Scientific and Technical Information, P.O. Box 62, Oak Ridge, TN 37831

112-170. Given distribution as shown in DOE/OSTI-4500, Magnetic Fusion Energy (Category Distribution UC-426, Experimental Plasma Physics) 


\section{Doe I}
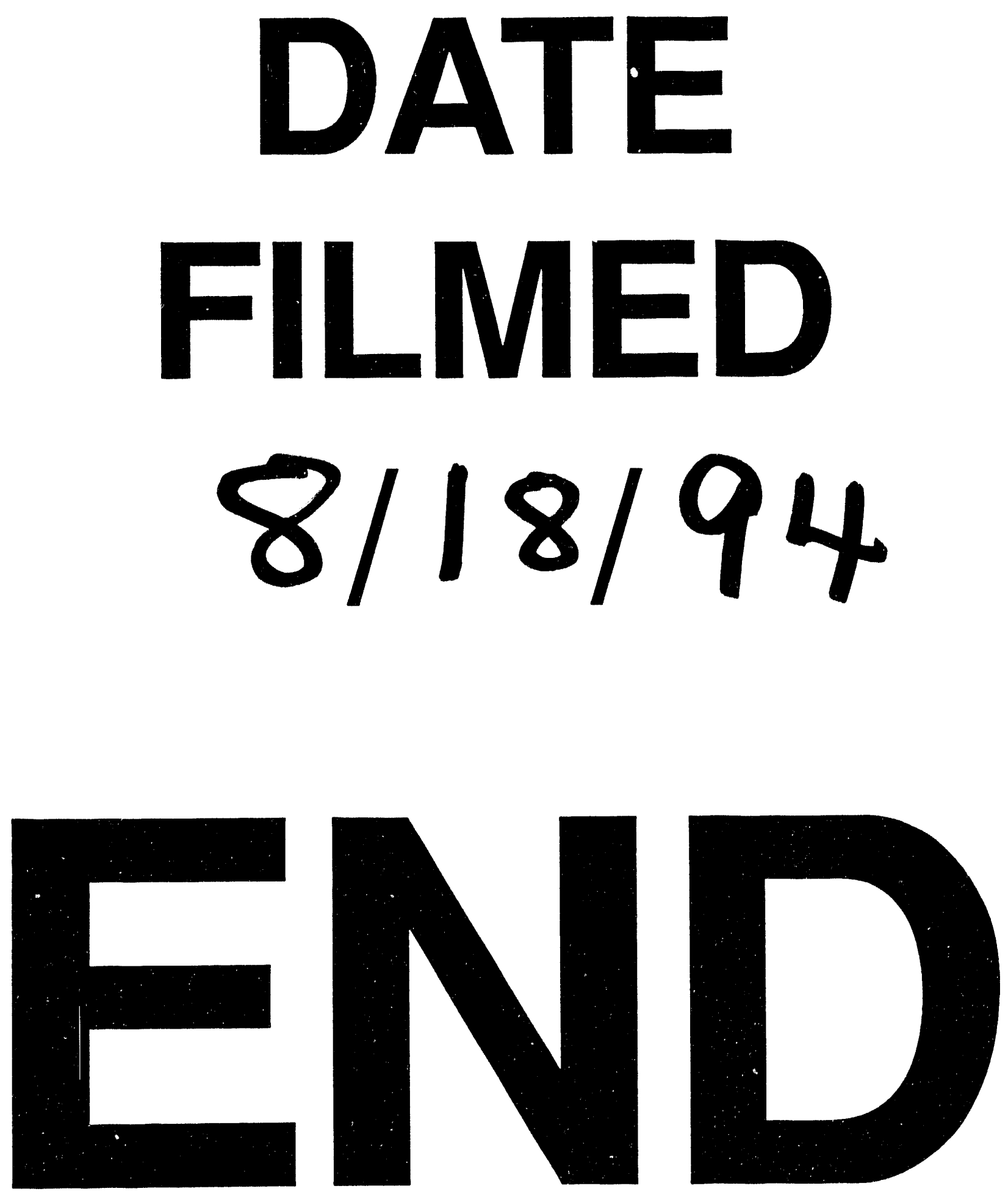
